A lumped model for rotational modes in periodic solid composites

This content has been downloaded from IOPscience. Please scroll down to see the full text. 2013 EPL 10426001

(http://iopscience.iop.org/0295-5075/104/2/26001)

View the table of contents for this issue, or go to the journal homepage for more

Download details:

IP Address: 109.171.137.234

This content was downloaded on 21/01/2015 at 12:02

Please note that terms and conditions apply. 


\title{
A lumped model for rotational modes in periodic solid composites
}

\author{
Pai Peng, Sharefa Asiri, Xiujuan Zhang, Yan Li ${ }^{(a)}$ and Ying Wu ${ }^{(b)}$ \\ Division of Computer, Electrical and Mathematical Sciences and Engineering, King Abdullah University of Science \\ and Technology (KAUST) - Thuwal 23955-6900, Saudi Arabia
}

received 21 June 2013; accepted in final form 15 October 2013

published online 8 November 2013

PACS 62.30.+d - Mechanical and acoustical properties of condensed matter: Mechanical and elastic waves; vibrations

PACS $62.65 .+\mathrm{k}-$ Acoustical properties of solids

PACS $62.20 .-\mathrm{x}$ - Mechanical properties of solids

\begin{abstract}
We present a lumped model to study the rotational modes in a type of two-dimensional periodic solid composites comprised of a square array of rubber-coated steel cylinders embedded in an epoxy matrix. The model captures the physical essence of rotational modes in such systems for various combinations of material parameters, and, therefore it is able to describe the transition behaviour when the system is gradually adjusted from an elastic metamaterial to an elastic phononic crystal. From the model, we can define a transition zone which separates the typical elastic metamaterials and the phononic crystals.
\end{abstract}

Copyright (C) EPLA, 2013

Introduction. - Metamaterials and photonic/phononic crystals are two typical types of artificial materials that are developed to control elastic, acoustic and/or electromagnetic waves [1-18]. One of the key distinctions [1,17] between a metamaterial and a photonic/phononic crystal is that metamaterials usually contain resonant structures to generate unique properties at the resonance frequencies $[2-8]$, whereas phononic/photonic crystals attribute their properties primarily to Bragg scattering [11-15]. Though the underlying mechanisms seem to be different, in some cases they bear the same interesting properties, for example, band gaps $[2-5,11-13]$ and negative refraction $[9,14-16]$. It is not always trivial to distinguish a metamaterial from a photonic or phononic crystal especially when both of them are made of periodic structures and their working frequencies are not separated far away [19]. In fact, some metamaterials can be converted to photonic or phononic crystals and vice versa, by gradually tuning the material parameters $[1,20]$. Smooth transition associated with an intermediate region has been found, which shows that there exists no clear boundary between a metamaterial and a phononic crystal.

Recently, elastic metamaterials (EMMs) and elastic phononic crystals (EPCs), whose host media are both

\footnotetext{
(a) Present address: Department of Physics, South China University of Technology - Guangzhou 510641, PRC.

(b) E-mail: ying. wu@kaust.edu.sa
}

solids, have attracted considerable attention due to the inherent rich physics [8-10,20-22]. For EMMs, a representative example is the so-called locally resonant material whose unit cell is a rubber-coated lead sphere embedded in an epoxy host [20]. Such EMMs can produce large band gap at very low frequencies. For EPC, one typical case is iron cylinders arranged periodically in epoxy [22], which gives common features of a phononic crystal such as Bragg band gap. In addition to their signature properties, both of these EMMs and EPCs support local rotation $[9,10,18,21-28]$, a unique property that belongs to solid composites. Rotational modes have been widely studied in granular crystals [23,24], EMMs and EPCs [25-28]. Liu et al. used continuum theories to study rotatable microstructures containing chiral lattice $[25,26]$. Bigoni et al. developed an asymptotic analysis to study the rotational motion of a type of locally resonant structure involving arrays of structured coated scatterers [27]. Most of these studies [23-28] are focused on the artificial structures with discretized components. Very recently, we proposed a simple lumped model to study the rotational motions in a kind of two-dimensional continuous EPCs [22]. Although the model gives a clear understanding of the rotational motion and successfully reproduces the three lowest bands associated with rotational and transverse modes, it fails in describing the similar motions in EMMs, because the scatterers in EMMs are composed of heavy cores coated with soft layers, which cannot be well modeled as rigid cylinders. 


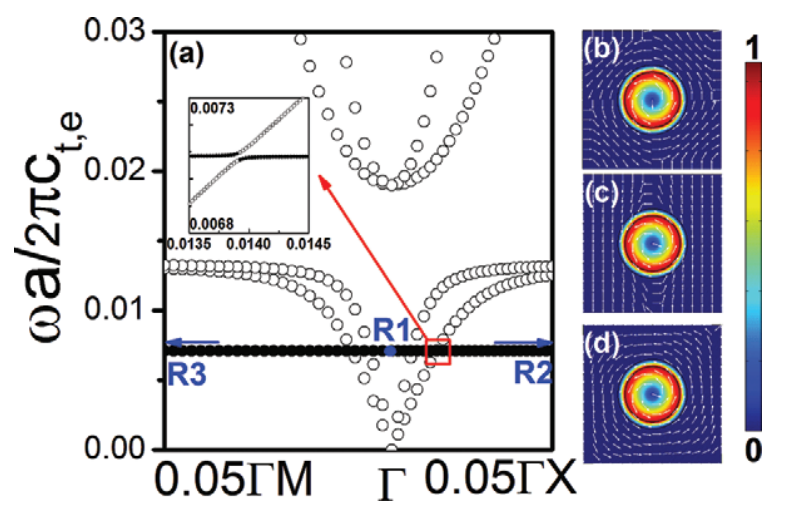

Fig. 1: (Colour on-line) (a) Band structure of the twodimensional metamaterials composed of a square array of steel cylinders coated with a layer of silicone rubber embedded in epoxy. One flat band is highlighted by solid circles. The inset illustrates an enlarged view of the gap induced by the flat band. Panels (b), (c) and (d) are the displacement field distributions of the eigenmodes $R 1, R 2$ and $R 3$ on the flat band at the $\Gamma, X$ and $M$ points, respectively. Dark red and dark blue correspond to one and zero of the normalized magnitude, respectively, and thin arrows indicate directions.

In this paper, we establish a new lumped model (NLM) for the rotational motions in both EMMs and EPCs. The model is capable of characterizing the key features of the rotational motion in both the EMMs and EPCs as well as the transition process between them when the parameters are gradually altered. It shows that although there is no clear boundary between the two systems, the relations between the two systems can be explained by the NLM. The paper is organized as follows. In the second section, we give a simple review for the band structure of $2 \mathrm{D}$ EMM, and in the third section we build a new lumped model to study the rotational modes of the system. We explain the transition states between the EMMs and the EPCs in the fourth section and conclude in the final section.

Rotational modes in elastic metamaterials. - The two-dimensional EMM studied here is a square array of steel cylinders coated with a thin layer of silicone rubber and embedded in epoxy. The inner and outer radii of the coating layer are $R=0.2 a$ and $R^{\prime}=0.25 a$, respectively ( $a$ is the lattice constant). The parameters of materials used are: $\rho_{e}=1180 \mathrm{~kg} / \mathrm{m}^{3}, \lambda_{e}=4.4 \times 10^{9} \mathrm{~N} / \mathrm{m}^{2}$ and $\mu_{e}=1.6 \times$ $10^{9} \mathrm{~N} / \mathrm{m}^{2}$ for epoxy; $\rho_{r}=1300 \mathrm{~kg} / \mathrm{m}^{3}, \lambda_{r}=6 \times 10^{5} \mathrm{~N} / \mathrm{m}^{2}$ and $\mu_{r}=4 \times 10^{4} \mathrm{~N} / \mathrm{m}^{2}$ for rubber; $\rho_{s}=7900 \mathrm{~kg} / \mathrm{m}^{3}$, $\lambda_{s}=1 \times 10^{11} \mathrm{~N} / \mathrm{m}^{2}$ and $\mu_{s}=8.1 \times 10^{10} \mathrm{~N} / \mathrm{m}^{2}$ for steel, respectively, where $\rho$ is mass density, and $\lambda$ and $\mu$ are Lame constants.

The band structures of such an EMM are shown in fig. 1(a), which are computed by COMSOL Multiphysics, a commercial package based on the finite-element method. The band structures display a complete band gap for both longitudinal and transverse waves from normalized frequency $\omega a / 2 \pi c_{t, e}=0.013$ to $\omega a / 2 \pi c_{t, e}=0.019$, where $c_{t, e}$ is the transvers wave velocity in matrix. The gap is

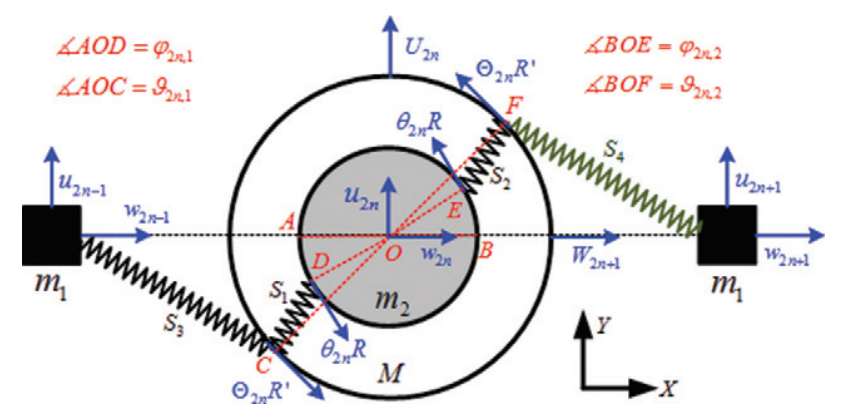

Fig. 2: (Colour on-line) A schematic of the new lumped model. The solid black squares indicate the matrix in one unit with mass $m_{1}$, and the gray disk indicates the finite-sized cylindrical steel with mass $m_{2}$. Springs $S 3$ and $S 4(S 1$ and $S 2)$ with a spring constant $K_{1}\left(K_{2}\right)$ present the interacting forces induced by deformations in matrix (rubber). The springs are connected to arbitrarily points, denoted as $C, D, E$ and $F$, where the $\varphi_{2 n, 1}, \varphi_{2 n, 2}, \vartheta_{2 n, 1}$ and $\vartheta_{2 n, 2}$ are the polar angles. The black circle (between $S 1$ and $S 3$ ) is the massless middlelayer $M$. Blue arrows denote the displacements of rotational and translational motions, where $\theta_{2 n}$ and $\Theta_{2 n}$ are the rotation angles of $m_{2}$ and $M$, respectively.

induced by dipolar resonance [3,5]. Below this band gap, there is a flat band, highlighted by solid circles, across the two branches. In fact, from an enlarged view shown in fig. 1(a), it can be seen that this flat band intersect the higher branches (longitudinal) and causes a tiny band gap when it goes through the lower branch (transverse). To better understand this flat band, we choose several highsymmetry points in the reduced Brillouin zone and plot their corresponding eigenstates. Figures 1(b), (c), and (d) show the displacement fields of the eigenmodes $R 1, R 2$ and $R 3$ (corresponding eigenfrequencies are $\omega_{R 1}, \omega_{R 2}$ and $\omega_{R 3}$ ) on the flat band at $\Gamma, X$ and $M$ points, respectively. All of these figures exhibit strong rotational motion concentrated on the inclusion. These localized states account for the flat band as the coupling between the states is very weak. By carefully examining the eigenfrequencies at these highsymmetry points, we also notice the flat band is not exactly non-dispersive: the eigenfrequency at the $\Gamma$-point is slightly higher than that at the $X$-point. The flat band and the rotational states described here cannot be well described by the lumped model developed earlier in ref. [22], where the scatterers are treated as rigid cylinders and cannot reflect the significant distortion of the rubber layer.

New lumped model. - To correctly describe the rotational modes mentioned in the last section, we build a NLM as shown in fig. 2. The NLM is established based on a lumped-mass system composed of two components for the waves propagating along the $x$-direction. The two components are matrix, which is modeled as a point mass $m_{1}$, and the iron cylinder with rubber coating, which is modeled as a scatterer with mass $m_{2}$. The restoring forces induced by deformations of the matrix are modeled as massless "continuous" springs [22,29] with spring constant $K_{1}$. Up to now, this model is exactly the same 
as the lumped model derived for EPCs [22]. However, for EMMs, the scatterer is no longer as simple as a rigid object. It has internal degree of freedoms. Since the deformation is highly concentrated on the thin rubber layer, and the iron cylinder is heavy, we model the scatterer as a finite-size cylinder with mass $m_{2}$ and moment of inertia $I_{2}=m_{2} R^{2} / 2$, connected to a massless spring with spring constant $K_{2}$. The choice of the roles for each component can be justified by the following facts. First, the mass of rubber is much smaller than that of the other materials. In this case, the ratio of masses of epoxy, rubber and steel is $m_{e}: m_{r}: m_{s} \approx 11.37: 1: 11.9$; second, the moduli of steel are of orders of magnitudes higher than both rubber and epoxy. Thus, under the same restoring force, the deformation of iron is negligible compared to the other two.

In order to capture the rotational and translational movements, we can set that the masses $m_{1}$ and $m_{2}$ are rotatable and moveable [22]. As shown in fig. 2, the vertical (horizontal) displacements of the masses $m_{1}$ and $m_{2}$ are $u_{2 n-1}\left(w_{2 n-1}\right)$ and $u_{2 n}\left(w_{2 n}\right)$, respectively, where $n$ is the index of the unit cell, and the rotation angle of mass $m_{2}$ is $\theta_{2 n}$. In particular, there exists a massless middle layer with radius $R^{\prime}$ (denoted by $M$ as shown in fig. 2) connected to the two kinds of springs. This middle layer delineates the boundary of the scatterer and is used to describe the displacement on the interface between the scatterer and the host. Its vertical (horizontal) displacement is $U_{2 n}\left(W_{2 n}\right)$ and rotation angle is $\Theta_{2 n}$. When the coating layer is thin, the radius $R^{\prime}$ can be approximated by $R$.

The relative motions between different components cause the restoring forces, which are demonstrated by springs (labeled as $S 1-S 4$ ) shown in fig. 2. We take one spring $S 4$ (highlighted by the green color) connected to the middle-layer $M$ on the mass $m_{1}$ as an example to calculate the restoring force. The force (exerted on $M$ ) comes from two sources: the rotational movement of the scatterer and the relative translational movement between the scatterer and $m_{1}$. The rotational-movements-induced force is $\vec{F}_{2 n, S 4}^{e, r}=-K_{1}\left(-\Theta_{2 n} R \sin \vartheta_{2 n, 2} \hat{x}+\Theta_{2 n} R \cos \vartheta_{2 n, 2} \hat{y}\right)$, where $\vartheta_{2 n, 2}$ is the polar angle as shown in fig. 2. Here we assume that the average force of all the "continuous" springs can be expressed in terms of an integral:

$$
\vec{F}_{2 n, S 4}^{r}=\int_{-\pi / 2}^{\pi / 2} \vec{F}_{2 n, S 4}^{e, r} \mathrm{~d} \vartheta_{2 n, 2} / \pi=2 K_{1} \Theta_{2 n} R / \pi \hat{y} .
$$

The horizontal component is zero, which means the rotational movements of the scatterers do not provide net forces along the horizontal direction on its adjacent matrices. Similar to the rotational-movement-induced force, the translational-movement-induced force can be written as $\vec{F}_{2 n, S 4}^{u}=-K_{1}\left(W_{2 n}-w_{2 n+1}\right) \hat{x}-K_{1}\left(U_{2 n}-u_{2 n+1}\right) \hat{y}$. The net restoring force (provided by $S 4$ and exerted on $M$ ) is the sum of the two parts, i.e., $\vec{F}_{2 n, S 4}=\vec{F}_{2 n, S 4}^{r}+\vec{F}_{2 n, S 4}^{u}$. The restoring forces also provide torques exerted on a rotatable object such as the scatters. The two portions of the torques induced by relatively rotational and translational movements of masses and exerted on $M$ can be obtained as $\vec{T}_{2 n, S 4}^{r}=-K_{1} \Theta_{2 n} R^{2} \hat{z}$ and $\vec{T}_{2 n, S 4}^{u}=$ $-2 K_{1} R\left(U_{2 n}-u_{2 n+1}\right) / \pi \hat{z}$, respectively, and the net torques is $\vec{T}_{2 n, S 4}=\vec{T}_{2 n, S 4}^{r}+\vec{T}_{2 n, S 4}^{u}$. The expressions of other interaction forces and torques can be obtained in a similar way (see the appendix). According to Newton's second law, we can get the following equations of motion:

$$
\begin{aligned}
& m_{1} \ddot{u}_{2 n-1}=K_{1}\left(-2 u_{2 n-1}+U_{2 n}+U_{2(n-1)}\right) \\
& \quad+2 K_{1} R\left(\Theta_{2(n-1)}-\Theta_{2 n}\right) / \pi \\
& m_{1} \ddot{w}_{2 n-1}=K_{1}\left(-2 w_{2 n-1}+W_{2 n}+W_{2(n-1)}\right) \\
& m_{2} \ddot{u}_{2 n}=2 K_{2}\left(U_{2 n}-u_{2 n}\right) \\
& m_{2} \ddot{w}_{2 n}=2 K_{2}\left(W_{2 n}-w_{2 n}\right) \\
& I \ddot{\theta}_{2 n}=2 K_{2} R^{2}\left(\Theta_{2 n}-\theta_{2 n}\right) \\
& 0=-2 K_{2}\left(U_{2 n}-u_{2 n}\right)-K_{1}\left(2 U_{2 n}-u_{2 n-1}-u_{2 n+1}\right), \\
& 0=-2 K_{2} R^{2}\left(\Theta_{2 n}-\theta_{2 n}\right)-2 K_{1} \Theta_{2 n} R^{2} \\
& \quad-2 K_{1} R\left(u_{2 n-1}-u_{2 n+1}\right) / \pi \\
& 0=-2 K_{2}\left(W_{2 n}-w_{2 n}\right)-K_{1}\left(2 W_{2 n}-w_{2 n-1}-w_{2 n+1}\right)
\end{aligned}
$$

where the first and second ones are derived for $m_{1}$, the third, fourth and fifth equations are for the translational and rotational motions of $m_{2}$, and the last three account for the balance of both forces and torques on the middlelayer $M$. The horizontal displacements are decoupled from both the vertical and rotational movements (see the appendix). It is, therefore, reasonable to ignore the horizontal movements in our study of the rotational modes. By supposing the vibration is time harmonic and invoking the Bloch theorem ( $q$ is the Bloch wave vector), we can obtain the secular equation as following:

$$
\text { see eq. (3) on the next page }
$$

While the expression of dispersion relation is complicated, solutions at high-symmetry points can be analytically solved as

$$
\begin{aligned}
& \begin{cases}\omega_{\Gamma 1} & =0, \\
\omega_{\Gamma 2} & =\sqrt{2 \bar{K} \frac{R^{2}}{I_{2}}}, \\
\omega_{\Gamma 3} & =\sqrt{2 \bar{K} \frac{m_{1}+m_{2}}{m_{1} m_{2}}},\end{cases} \\
& \left\{\begin{array}{l}
\omega_{X 1}=\sqrt{2 \bar{K} \frac{1}{m_{2}}}, \\
\omega_{X 2}=\sqrt{2 \bar{K} \frac{R^{2}}{I_{2}}-\Delta,} \\
\omega_{X 3}=\sqrt{2 K_{1} \frac{1}{m_{1}}\left(1-\frac{4 \bar{K}}{\pi^{2} K_{2}}\right)+\Delta}
\end{array}\right.
\end{aligned}
$$

for the $\Gamma$-point $(q=0)$ and $X$-point $(q=\pi / a)$, respectively, where $\bar{K}=1 /\left(1 / K_{1}+1 / K_{2}\right)$ and $\Delta$ is a positive quantity $\Delta=2 \bar{K}\left(\sqrt{a^{2}+b}-a\right)$ with $a=$ 


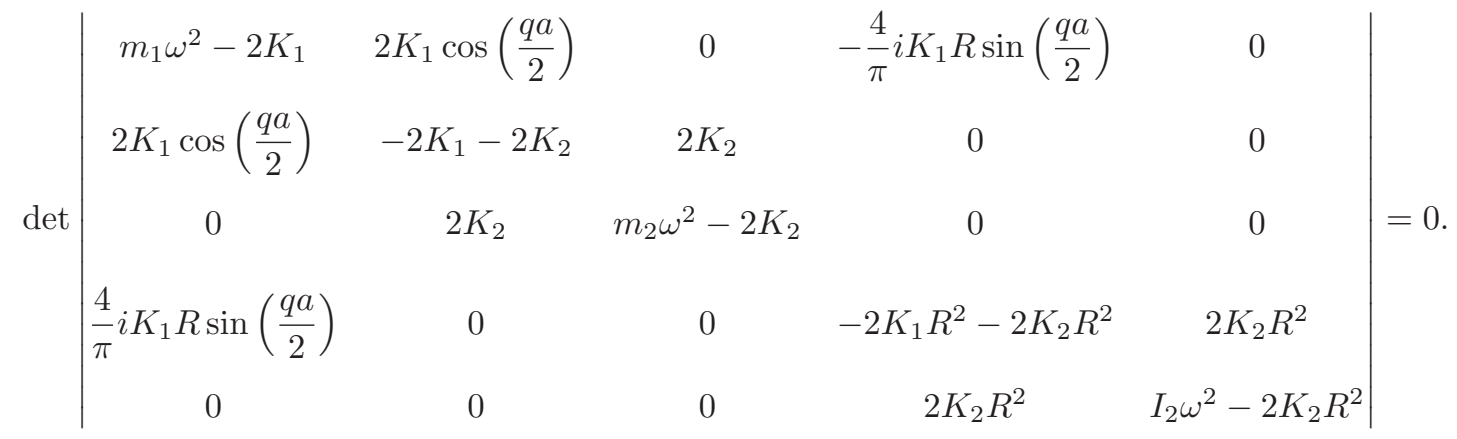

$\left(I_{2}-R^{2} m_{1}\right) /\left(2 m_{1} I_{2}\right)+K_{1}\left(1-4 / \pi^{2}\right) /\left(2 K_{2} m_{1}\right)$ and $b=$ $4 R^{2} /\left(\pi^{2} m_{1} I_{2}\right)$. Obviously, $\omega_{\Gamma 2}$ is the resonant frequency of a harmonic rotating oscillator and $\omega_{\Gamma 3}$ is the typical frequency of an optical branch of a di-atomic chain. The eigenfrequencies at the $X$-point of the reduced Brillouin zone may be interpreted as resonant frequencies of an acoustic branch of a di-atomic chain $\left(\omega_{X 1}\right)$ and rotationtranslation hybrid states $\left(\omega_{X 2}\right.$ and $\left.\omega_{X 3}\right)$.

In the NLM, $K_{1}$ and $K_{2}$ are associated with the moduli of epoxy and rubber, respectively, while the geometry parameters are fixed. This is reasonable as the moduli in the real system are incorporated into the spring constants adopted in the NLM. The eigenfrequencies given by eqs. (4) hold for a general solid composite with a square array of cylindrical solid inclusions. It indeed agrees with the phenomenon observed for both EMMs and EPCs. If the coating layer is the softest among all the materials, which is the case shown in fig. $1, K_{2}$ in the NLM is much smaller than $K_{1}$ so that $\bar{K}$ approximately equals $K_{2}$. The eigenfrequencies of the two translational modes are $\omega_{X 1}=\sqrt{2 K_{2} / m_{2}}$ and $\omega_{\Gamma 3}=\sqrt{2 K_{2}\left(1 / m_{1}+1 / m_{2}\right)}$, which is similar to those studied in ref. [30]. These two frequencies denote a band gap induced by the dipolar resonance $[4,30]$. For the rotation-related states, $\omega_{\Gamma 2}$ and $\omega_{X 2}$ in the NLM are associated with the frequencies $\omega_{R 1}$ and $\omega_{R 2}$ in the real system, respectively. The value of $\omega_{\Gamma 2}$ is very close to that of $\omega_{X 2}$ because the term $\Delta$ in the expression of $\omega_{X 2}$ tends to vanish. This is because $\Delta$ is on the order of $\bar{K} b / a$ as $a$ is much bigger than $b$, which is a result of $K_{2} \ll K_{1}$. This explains the origin of the "flat band" shown in fig. 1. If the coating layer is steel rather than rubber, we have an EPC. While the coating layer becomes stiffer, in the limit of $K_{2} \gg K_{1}, \bar{K}$ approximately equals $K_{1}$ and the NLM recovers the results developed for EPCs [22].

EMM to EPC transition. - The rotational modes in both EMMs and EPCs can be described by the NLM, so the NLM would be a good method to study the intermediate systems between the EMMs and EPCs. Here we consider the EMM studied earlier. The intermediate systems can be realized by gradually increasing the stiffness and mass density of the rubber until the parameters reach those values of steel. For simplicity, we introduce a single parameter $\mu_{x} \in\left[\mu_{r}, \mu_{s}\right]$ to characterize the intermediate systems in such a way that $\rho_{x}=\left(\rho_{s}-\rho_{r}\right)\left(\mu_{x} / \mu_{r}-\right.$ 1) $\mu_{r} /\left(\mu_{s}-\mu_{r}\right)+\rho_{r}, \lambda_{x}=\left(\lambda_{s}-\lambda_{r}\right)\left(\mu_{x} / \mu_{r}-1\right) \mu_{r} /\left(\mu_{s}-\right.$ $\left.\mu_{r}\right)+\lambda_{r}$ and $\mu_{x}=\mu_{x}$. It can be seen that when $\mu_{x}=\mu_{r}$ or $\mu_{s}$, the other two quantities also take their corresponding values of rubber or steel. For each $\mu_{x}$, we substitute the corresponding material parameters into the coating layer of the EMM and compute its band structure, from which we can get the eigenfrequencies $\omega_{R 1}$ and $\omega_{R 2}$ of eigenmodes $R 1$ and $R 2$, respectively. We plot two quantities, $\omega_{R 1}$ and $\omega_{\Delta}=\omega_{R 1}-\omega_{R 2}$, as functions of $\mu_{x}$ in black solid curves in figs. 3(a) and (b), respectively, which shows that as $\mu_{x}$ increases both $\omega_{R 1}$ and $\omega_{\Delta}$ increase (linearly in logarithmic scale) before their values saturate at $\mu_{x}=\mu_{e}$. When $\mu_{x}$ is greater than $\mu_{e}$, both $\omega_{R 1}$ and $\omega_{\Delta}$ can be relatively regarded as constants.

The above transition behaviors can also be characterized by the NLM. We use $\Delta$ instead of $\omega_{\Delta}$ to describe the bandwidth, and $K_{2}$ is associated with $\mu_{x}$. Corresponding to the real systems, figs. $3(\mathrm{c})$ and (d) show the dependence of $\omega_{\Gamma 2}$ and $\Delta$ on $K_{2}$, for systems with different values of mass $m_{2}$ but fixed values of $m_{1}=1, R=0.2, a=1$ and $K_{1}=1$. This group of curves display similar behaviors as those shown in figs. $3(\mathrm{a})$ and (b), but the turning point is $K_{2}=K_{1}$ rather than $\mu_{x}=\mu_{e}$. From eqs. (4) which is obtained from the NLM, we can write $\omega_{\Gamma 2}$ as

$$
\log \left(\omega_{\Gamma 2}\right)=\frac{1}{2} \log (\bar{K})+\frac{1}{2} \log \left(\frac{4}{m_{2}}\right) .
$$

The expression tells us when $K_{2}$ is much smaller than $K_{1}$, which is the case of an EMM with very soft coating layer, the first term on the right-hand side is approximately $\log \left(K_{2}\right) / 2$ so that $\log \left(\omega_{\Gamma 2}\right)$ depends linearly on $\log \left(K_{2}\right)$. The NLM gives the slopes which are 0.5 and 1.52 for $\omega_{R 1}$ and $\omega_{\Delta}$ demonstrated as red straight lines shown in figs. 3(a) and (b), respectively. Here the parameters used in the NLM are $m_{1}=\rho_{e}\left(a^{2}-\pi R^{\prime 2}\right), m_{2}=\rho_{s} \pi R^{2}$ and $K_{1}=10^{4} K_{2}=\mu_{e}$. The red lines agree well with the curves obtained from the real systems. On the other hand, when $K_{2}$ is much larger than $K_{1}$, we have $\bar{K} \approx K_{1}$, and $\omega_{\Gamma 2}$ becomes independent of $K_{2}$. It means if the coating layer is much harder than the matrix, the frequency $\omega_{R 1}$ 

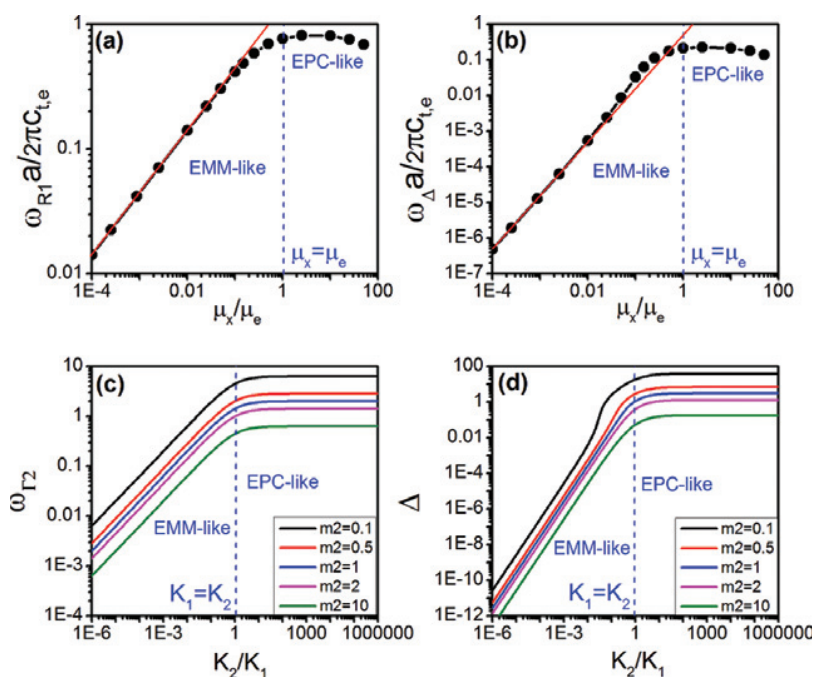

Fig. 3: (Colour on-line) (a) Normalized $\omega_{R 1}$ and (b) $\omega_{\Delta}$ both obtained from the band structures of real systems are plotted as a function of $\mu_{x}$, which is the stiffness of the coating layer. Red lines denote the slope predicted by the NLM. The dependence of $\omega_{\Gamma 2}$ (c) and $\Delta$ (d) on $K_{2}$ is obtained from eqs. (4), for systems with different values of mass $m_{2}$ but fixed values of $m_{1}=1$, $R=0.2$ and $K_{1}=1$.

becomes independent of $\mu_{x}$. This is the reason why $\omega_{R 1}$ is relatively a constant when $\mu_{x} \gg \mu_{e}$. The properties of $\omega_{\Delta}$, as plotted in fig. $3(\mathrm{~b})$, are similar to those of $\omega_{\Gamma 2}$. This may come from the fact that the coefficient $\sqrt{2 \bar{K}}$ of $\Delta$ is the same as that of $\omega_{\Gamma 2}$.

As shown in figs. 3(a) and (b), the transitions states can be divided into two parts. When $\mu_{x}$ is smaller than $\mu_{e}$, the system is closer to an EMM. While the rubber is the softest among all the materials, the deformations are concentrated on the rubber. As a result, the rotational modes are localized in the scatterers. In this case, the resonant frequency $\omega_{R 1}$ can be very low, and the band induced by coupled rotational-translational modes is very "flat" (as shown in fig. 1). On the contrary, when $\mu_{x}$ is greater than $\mu_{e}$, the system behaves more like an EPC. The system properties are no longer sensitive to $\mu_{x}$. The transition will occur in the vicinity of $\mu_{e}$.

In the case of a hard coating layer $\left(\mu_{x} \gg \mu_{e}\right)$, we notice that $\omega_{R 1}$ and $\omega_{\Delta}$ both slightly decreased with the growth of $\mu_{x}$. This can be interpreted as changes of $m_{2}$. While the coating layer gets harder, it also gets heavier according to the constraint relation mentioned earlier. Under this condition the rubber cannot be regarded as massless spring in the EPCs case. Here $m_{2}$ should include the mass of rubber, which get $m_{2}$ increased. Figures $3(\mathrm{c})$ and (d) show the results obtained from the NLM: both $\omega_{\Gamma 2}$ and $\Delta$ decrease with the increase of $m_{2}$.

Conclusions and discussions. - In this work, we build a NLM to study the rotational modes in both EMMs and EPCs. The EMMs differentiate from the EPCs by an extra soft coating layer around the cylindrical scat- terer. The NLM is able to produce, respectively, flat and dispersive bands induced by the coupled rotationaltransverse motion for EMMs and EPCs. It also captures the key feature of the transition from an EMM to an EPC by gradually altering the material parameters of the coating.

As introduced in ref. [17], one of the key differences between EMMs and EPCs should be the dependence of the resonant frequency on the geometry, including lattice parameters and incidence directions. This difference can be explained by the NLM and attributed to the key function $\bar{K}=1 /\left(1 / K_{1}+1 / K_{2}\right)$. The change in $\bar{K}$ is more sensitive to the change in the smaller one of $K_{1}$ and $K_{2}$. In real physical systems, spring constants are related to the material and geometry parameters. That means the lattice constant, pertained to the geometry of the matrix, is associated with $K_{1}$. To alter the lattice constant changes $K_{1}$, which makes very small contributions to $\bar{K}$ in the case of an $\operatorname{EMM}\left(K_{2} \ll K_{1}\right)$. So the resonance frequency (i.e. $\omega_{\Gamma 2}$ ) and some other properties of the system, such as $\omega_{\Delta}$, are sensitive to the parameters of the rubber but are insensitive to the matrix. This may give a reference to define the transition states. From the perspective of the NLM, the transition occurs at $K_{1}=K_{2}$.

\section{APPENDIX}

The restoring forces and torques $(\vec{T}=\vec{L} \times \vec{F}$, with $\vec{L}$ the corresponding lever arm vector) for each spring exerted on $M$ are

$$
\begin{aligned}
\vec{F}_{2 n, S 1}= & -K_{2}\left(W_{2 n}-w_{2 n}\right) \hat{x} \\
& -\left[K_{2}\left(U_{2 n}-u_{2 n}\right)-2 K_{2}\left(\Theta_{2 n}-\theta_{2 n}\right) R / \pi\right] \hat{y}, \\
\vec{F}_{2 n, S 2}= & -K_{2}\left(W_{2 n}-w_{2 n}\right) \hat{x} \\
& -\left[K_{2}\left(U_{2 n}-u_{2 n}\right)+2 K_{2}\left(\Theta_{2 n}-\theta_{2 n}\right) R / \pi\right] \hat{y}, \\
\vec{F}_{2 n, S 3}= & -K_{1}\left(W_{2 n}-w_{2 n-1}\right) \hat{x} \\
& -\left[K_{1}\left(U_{2 n}-u_{2 n-1}\right)-2 K_{1} \Theta_{2 n} R / \pi\right] \hat{y}, \\
\vec{F}_{2 n, S 4}= & -K_{1}\left(W_{2 n}-w_{2 n+1}\right) \hat{x} \\
& -\left[K_{1}\left(U_{2 n}-u_{2 n+1}\right)+2 K_{1} \Theta_{2 n} R / \pi\right] \hat{y}
\end{aligned}
$$

and

$$
\begin{aligned}
& \vec{T}_{2 n, S 1}=-\left[K_{2}\left(\Theta_{2 n}-\theta_{2 n}\right) R^{2}-2 K_{2}\left(U_{2 n}-u_{2 n}\right) R / \pi\right] \hat{z} \\
& \vec{T}_{2 n, S 2}=-\left[K_{2}\left(\Theta_{2 n}-\theta_{2 n}\right) R^{2}+2 K_{2}\left(U_{2 n}-u_{2 n}\right) R / \pi\right] \hat{z} \\
& \vec{T}_{2 n, S 3}=-\left[K_{1} \Theta_{2 n} R^{2}-2 K_{1}\left(U_{2 n}-u_{2 n-1}\right) R / \pi\right] \hat{z} \\
& \vec{T}_{2 n, S 4}=-\left[K_{1} \Theta_{2 n} R^{2}+2 K_{1}\left(U_{2 n}-u_{2 n+1}\right) R / \pi\right] \hat{z}
\end{aligned}
$$

respectively. We note that integrations have been used (i.e., eq. (1)) to sum up the forces and torques on the interfaces, and the terms including a sine function vanish due to the symmetry. The net force and torque exerted on $M$ can be obtained as

$$
\begin{aligned}
\vec{F}_{2 n}^{M}= & \vec{F}_{2 n, S 1}+\vec{F}_{2 n, S 2}+\vec{F}_{2 n, S 3}+\vec{F}_{2 n, S 4} \\
= & {\left[-2 K_{2}\left(W_{2 n}-w_{2 n}\right)-K_{1}\left(2 W_{2 n}-w_{2 n-1}-w_{2 n+1}\right)\right] \hat{x} } \\
& +\left[-2 K_{2}\left(U_{2 n}-u_{2 n}\right)-K_{1}\left(2 U_{2 n}-u_{2 n-1}-u_{2 n+1}\right)\right] \hat{y}
\end{aligned}
$$




$$
\begin{aligned}
\vec{T}_{2 n}^{M}= & \vec{T}_{2 n, S 1}+\vec{T}_{2 n, S 2}+\vec{T}_{2 n, S 3}+\vec{T}_{2 n, S 4} \\
= & {\left[-2 K_{2}\left(\Theta_{2 n}-\theta_{2 n}\right) R^{2}-2 K_{1} \Theta_{2 n} R^{2}\right.} \\
& \left.-2 K_{1}\left(u_{2 n-1}-u_{2 n+1}\right) R / \pi\right] \hat{z} .
\end{aligned}
$$

Similarly, the net force and torques exerted on $m_{2}$ are

$$
\begin{aligned}
\vec{F}_{2 n}^{m_{2}} & =-\vec{F}_{2 n, S 1}-\vec{F}_{2 n, S 2} \\
& =2 K_{2}\left(W_{2 n}-w_{2 n}\right) \hat{x}+2 K_{2}\left(U_{2 n}-u_{2 n}\right) \hat{y}, \\
\vec{T}_{2 n}^{m_{2}} & =-\vec{T}_{2 n, S 1}-\vec{T}_{2 n, S 2}=2 K_{2}\left(\Theta_{2 n}-\theta_{2 n}\right) R^{2} \hat{z} .
\end{aligned}
$$

The net force exerted on $m_{1}$ is

$$
\begin{aligned}
\vec{F}_{2 n-1}^{m_{1}}= & -\vec{F}_{2 n, S 3}-\vec{F}_{2(n-1), S 4} \\
= & K_{1}\left(-2 w_{2 n-1}+W_{2 n}+W_{2(n-1)}\right) \hat{x} \\
& +\left[K_{1}\left(-2 u_{2 n-1}+U_{2 n}+U_{2(n-1)}\right)\right. \\
& \left.+2 K_{1}\left(\Theta_{2(n-1)}-\Theta_{2 n}\right) R / \pi\right] \hat{y} .
\end{aligned}
$$

Thus, we can obtain the equations of motion as shown in eq. (2), from which we get the following expression:

$\left(\begin{array}{cccccccc}d_{11} & 0 & d_{13} & 0 & d_{15} & 0 & 0 & 0 \\ 0 & d_{22} & d_{23} & 0 & 0 & 0 & 0 & 0 \\ d_{13} & d_{23} & d_{33} & 0 & 0 & 0 & 0 & 0 \\ 0 & 0 & 0 & d_{44} & d_{45} & 0 & 0 & 0 \\ -d_{15} & 0 & 0 & d_{45} & d_{55} & 0 & 0 & 0 \\ 0 & 0 & 0 & 0 & 0 & d_{66} & 0 & d_{68} \\ 0 & 0 & 0 & 0 & 0 & 0 & d_{77} & d_{78} \\ 0 & 0 & 0 & 0 & 0 & d_{68} & d_{78} & d_{88}\end{array}\right)\left(\begin{array}{c}Y_{1} \\ Y_{2} \\ Y_{3} \\ O_{4} \\ O_{5} \\ X_{6} \\ X_{7} \\ X_{8}\end{array}\right)=0$

with $Y_{1}, Y_{2}, Y_{3}, O_{4}, O_{5}, X_{6}, X_{7}$ and $X_{8}$ denoting the arbitrary amplitudes for $u_{2 n-1}, u_{2 n}, U_{2 n}, \theta_{2 n}$, $\Theta_{2 n}, w_{2 n-1}, w_{2 n}$ and $W_{2 n}$, respectively (i.e., $u_{2 n-1}=$ $\left.Y_{1} e^{i q(2 n-1) a / 2} e^{i w t}\right)$. The non-zero terms $\left(d_{i j}\right)$ in the matrix are

$$
\begin{array}{llrl}
d_{11}=\omega^{2} m_{1}-2 K_{1}, & & d_{88}=-2 K_{2}-2 K_{1}, \\
d_{22}=\omega^{2} m_{2}-2 K_{2}, & & d_{13}=2 K_{1} \cos (q a / 2), \\
d_{33}=-2 K_{1}-2 K_{2}, & & d_{15}=-4 i K_{1} R \sin (q a / 2) / \pi, \\
d_{44}=\omega^{2} I-2 K_{2} R^{2}, & & d_{23}=2 K_{2}, \\
d_{55}=-2 K_{2} R^{2}-2 K_{1} R^{2}, & d_{45}=2 K_{2} R^{2}, \\
d_{66}=\omega^{2} m_{1}-2 K_{1}, & d_{68}=4 K_{1} \cos (q a / 2), \\
d_{77}=\omega^{2} m_{2}-2 K_{2}, & d_{78}=2 K_{2} .
\end{array}
$$

We can see that the terms $d_{15}$ and $-d_{15}$ represent the coupling between the rotational movements and the displacements on the $y$-direction. In contrast, the displacements on the $x$-direction are independent of the other two kinds of movements.

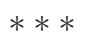

The work described here was supported by the KAUST Baseline Research Fund.

\section{REFERENCES}

[1] Ao X. Y. and Chan C. T., Phys. Rev. B, 80 (2009) 235118.
[2] FAng N., Xi D. J., XU J. Y., Ambati M., Srituravanich W., Sun C. and Zhang X., Nat. Mater., 5 (2006) 452.

[3] Liu Z. Y., Zhang X. X., MaO Y. W., Zhu Y. Y., Yang Z. Y., Chan C. T. and Sheng P., Science, 289 (2000) 1734 .

[4] Liu Z. Y., Chan C. T. and Sheng P., Phys. Rev. B, 71 (2005) 014103.

[5] Wu Y., Lai Y. and Zhang Z. Q., Phys. Rev. B, 76 (2007) 205313.

[6] Kafesaki M., Penciu R. S. and Economou E. N., Phys. Rev. Lett., 84 (2000) 6050.

[7] Li J. and Chan C. T., Phys. Rev. E, 70 (2004) 055602.

[8] Lai Y., Wu Y., Sheng P. and Zhang Z. Q., Nat. Mater., 10 (2011) 620.

[9] Liu X. N., Hu G. K., Huang G. L. and Sun C. T., Appl. Phys. Lett., 98 (2011) 251907.

[10] Wang G., Wen X. S., Wen J. H., Shao L. H. and LiU Y. Z., Phys. Rev. Lett., 93 (2004) 154302.

[11] Yang S. X., Page J. H., Liu Z. Y., Cowan M. L., Chan C. T. and Sheng P., Phys. Rev. Lett., 88 (2002) 104301.

[12] Torres M., de Espinosa F. R. M., Garcia-Pablos D. and Garcia N., Phys. Rev. Lett., 82 (1999) 3054.

[13] Martinezsala R., Sancho J., Sanchez J. V., Gomez V., Llinares J. and Meseguer F., Nature, 378 (1995) 241.

[14] Cubukcu E., Aydin K., Ozbay E., Foteinopoulou S. and Soukoulis C. M., Nature, 423 (2003) 604.

[15] Croenne C., Manga E. D., Morvan B., Tinel A., Dubus B., Vasseur J. and Hladky-Hennion A. C., Phys. Rev. B, 83 (2011) 054301.

[16] Pendry J. B., Phys. Rev. Lett., 85 (2000) 3966.

[17] Fok L., Ambati M. and Zhang X., MRS Bull., 33 (2008) 931.

[18] Zhao H. G., Liu Y. Z., Wang G., Wen J. H., Yu D. L., Han X. Y. and Wen X. S., Phys. Rev. B, 72 (2005) 012301.

[19] LiU L. and Hussein M. I., J. Appl. Mech. Trans. ASME, 79 (2012) 011003.

[20] Peng P., Qiu C. Y., Ding Y. Q., He Z. J., Yang H. and Liu Z. Y., Solid State Commun., 151 (2011) 400.

[21] Liu F. M., Lai Y., Huang X. Q. and Chan C. T., Phys. Rev. B, 84 (2011) 224113.

[22] Peng P., Mei J. and Wu Y., Phys. Rev. B, 86 (2012) 134304.

[23] Schwartz L. M., Johnson D. L. and Feng S., Phys. Rev. Lett., 52 (1984) 831.

[24] Tournat V., Perez-Arjona I., Merkel A., SanchezMorcillo V. and Gusev V., New J. Phys. 13 (2011).

[25] Liu X. N., Huang G. L. and Hu G. K., J. Mech. Phys. Solids, 60 (2012) 1907.

[26] Liu X. N., Hu G. K., Sun C. T. and Huang G. L., J. Sound Vib., 330 (2011) 2536.

[27] Bigoni D., Guenneau S., Movchan A. B. and Brun M., Phys. Rev. B, 87 (2013).

[28] Spadoni A., Ruzzene M., Gonella S. and Scarpa F., Wave Motion, 46 (2009) 435.

[29] Huang H. H. and Sun C. T., Mech. Mater., 46 (2012) 1.

[30] Milton G. W. and Willis J. R., Proc. R. Soc. London, Ser. A, 463 (2007) 855. 